\title{
MÉTODO EXPERIMENTAL PARA DETERMINAÇÃO DOS LIMITES DE EXPANSÃO DE LATAS DE TRÊS PEÇAS
}

\author{
Paulo Roberto Campissi ${ }^{1}$ \\ Luciano Pessanha Moreira ${ }^{2}$ \\ Eric Jeronimo de Oliveira Alvarez ${ }^{3}$ \\ Carlos Sérgio da Costa Viana ${ }^{4}$ \\ William Ribeiro dos Santos ${ }^{5}$
}

\begin{abstract}
Resumo
Este trabalho apresenta um novo método para avaliar a conformabilidade de folhas de flandres usadas na produção de latas de três peças por expansão mecânica. Os corpos de prova são tubos soldados com grade litografada de $2,5 \mathrm{~mm}$ para medição das deformações nas direções meridionais e circunferenciais. Foi projetado um ferramental específico fixando-se alguns parâmetros como número e largura das pinças de expansão. $\mathrm{O}$ aparato é adaptado a uma máquina Erichsen, na qual o deslocamento radial do ferramental é controlado por um sistema hidráulico. Os estados de deformação são obtidos variando-se os raios das ferramentas. Os ensaios de expansão foram realizados com folha de flandres de $0,18 \mathrm{~mm}$ até a fratura do tubo e, em seguida, foram obtidas as deformaçães com um programa de correlação de imagens digitais. O ferramental de expansão proposto possibilita obter deformações limites de folhas finas revestidas de um modo simples comparado com as técnicas de Curva Limite de Conformação (CLC). Portanto, este método torna-se muito útil para desafios de inovação tecnológica e reduções de custos no mercado global de embalagens metálicas.
\end{abstract}

Palavras-chave: Embalagem; Deformação; Conformação; Folha-de-flandres.

\section{EXPERIMENTAL METHOD FOR DETERMINING THE EXPANSION LIMITS OF THREE-PIECE CANS}

\begin{abstract}
This work presents a new method to determine the formability of thin tinplate metallic sheets used to produce three-piece cans by mechanical expansion. The specimens consist of seamed welded tubes wherein a $2.5 \mathrm{~mm}$ square grid was printed by lithography to measure the hoop and meridional strains. A specific expansion tooling was designed by keeping constant some parameters such as the number and the width of the tools. The device is adapted to an Erichsen testing machine, in which the tooling displacement is controlled by a hydraulic system. The strain states are achieved by varying the tooling radii. The expansion tests were performed with a tinplate sheet with $0.18 \mathrm{~mm}$ thickness up to the tube fracture and then, the strains were obtained with a digital image correlation software. The proposed mechanical expansion tooling allows for attaining limit strains of coated thin metal sheets in a simple way compared to the Forming Limit Curve testing techniques. Therefore, this new method turns to be very useful to the challenges of technological innovations and costs reduction in the global market of metal packaging.
\end{abstract}

Key words: Metal packaging; Strain; Forming; Metallic sheets.

\footnotetext{
' Eng. Metalúrgico, M.Sc., Companhia Siderúrgica Nacional, Rua 4, 33, Cep 27265-6I0, Volta Redonda, RJ, Brasil. E-mail: pcampissi@csn.com.br

${ }^{2}$ Eng. Mecânico, D.Sc., Universidade Federal Fluminense, Av. dos Trabalhadores, 420, Cep 27255-I25, Volta Redonda, RJ,Brasil.

E-mail: luciano.moreira@metal.eeimvr.uff.br

${ }^{3}$ Eng. Metalúrgico, Companhia Siderúrgica Nacional, Rua 4, 33, Cep 27265-610, Volta Redonda, RJ, Brasil. E-mail: eric.oliveira@csn.com.br

${ }^{4}$ Eng. Metalúrgico, PhD., Universidade Federal Fluminense, Av. dos Trabalhadores 420, Cep 27255-I25, Volta Redonda, RJ, Brasil.

E-mail:viana@metal.eeimvr.uff.br

${ }^{5}$ Administrador, Companhia Siderúrgica Nacional, Rua 4, 33, Cep 27265-610, Volta Redonda, RJ, Brasil. E-mail: williamr@csn.com.br
} 


\section{INTRODUÇÃO}

De acordo com pesquisas recentes, ${ }^{(1)}$ o consumo mundial de bebidas e produtos alimentícios acondicionados em embalagens vem crescendo a cada ano. Observa-se que $\circ$ acondicionamento difere de região para região no mundo segundo condições climáticas e disponibilidade de produtos no mercado. A variedade de produtos que têm a sua integridade preservada por uma embalagem cresceu de maneira exponencial, permitindo a produção em grande escala e redução de preços. No Brasil, ao ser feita uma análise do consumo nos últimos anos por tipo de material da embalagem obteve-se a seguinte distribuição:(1) polímeros $(5 / \%)$, celulose $(27 \%)$, metais (17\%) e vidro (5\%). Com o grande crescimento do mercado de embalagens e com o mercado consumidor cada vez mais exigente, foram desenvolvidas técnicas para atribuir formas aos corpos das latas, em princípio com geometrias cilíndricas por meio de algum processo de expansão, aumentando a atratividade e a funcionalidade da embalagem. Essa expansão pode ser obtida por métodos mecânicos, hidráulicos e pneumáticos. Esses processos já são utilizados em países como México, Japão, Alemanha, França, entre outros, sendo que, na maioria dos casos, a expansão é efetuada por método mecânico. No Brasil, em 200 I, a Indústria Bertol implantou em sua fábrica uma linha de expansão para embalagens de $320 \mathrm{~mL}$ com capacidade de produção de 500 latas/min. Em 2004, a Nestlé/Brasil também inovou com o lançamento de uma lata exclusiva para o leite condensado com uma produção de I,5 milhão de latas/mês. $O$ desenvolvimento dessa nova embalagem foi realizado em escala piloto, em uma máquina expansora instalada na Gerência de Desenvolvimentos da Companhia Siderúrgica Nacional (CSN). Logo, para que essas embalagens inovadoras sejam concebidas de forma ótima com vistas à produção em escala industrial, fez-se necessário o estabelecimento de uma nova metodologia para determinação dos limites de expansão de folhas metálicas.

$O$ presente trabalho tem por objetivo desenvolver uma metodologia para avaliar a capacidade de conformação de folhas metálicas com espessura nominal $\leq 0,40 \mathrm{~mm}$, utilizadas na produção por expansão mecânica de latas de três peças. Para tal, foi projetado um dispositivo de expansão mecânica de corpos de prova de folhas de flandres na forma de tubo soldado. A depuração de deformações resultantes dos ensaios de expansão foi realizada com um sistema automático de aquisição e análise de imagens digitais. Por fim, as deformações limites obtidas por expansão mecânica foram comparadas com previsões de um modelo de estricção localizada.

\section{MATERIAIS E MÉTODOS}

Para elaboração deste trabalho foram utilizadas folhas de flandres produzidas pela CSN via recozimento contínuo, de simples redução, com $0,18 \mathrm{~mm}$ de espessura, e revestimentos de $5,6 \mathrm{~g} / \mathrm{m}^{2}$ e $2,8 \mathrm{~g} / \mathrm{m}^{2}$ de estanho nas superfícies inferior e superior, que correspondem aos lados interno e externo da embalagem respectivamente.

\section{I Análises Químicas e Metalográficas}

As análises químicas com amostras na forma de chapa foram realizadas por espectrometria de emissão óptica, para determinação do percentual por peso dos elementos químicos: $\mathrm{Mn}, \mathrm{P}, \mathrm{Si}, \mathrm{Cu}, \mathrm{Cr}, \mathrm{Mo}, \mathrm{B}, \mathrm{Ti}$ e Al. As composições do percentual por peso de $\mathrm{C}, \mathrm{S}$ e $\mathrm{N}$ foram obtidas por meio de absorção atômica com amostras na forma de limalha. A análise metalográfica, sem ataque químico, foi realizada nas amostras para avaliar a sanidade interna em conformidade com o Método $\mathrm{A}$ da norma ASTM E 45.(2) O processo foi finalizado mediante análise metalográfica, com ataque químico por meio de solução Nital 3\%, nas amostras, para avaliar a microestrutura e o tamanho de grão ferrítico segundo a norma ASTM E- I | 2. (3) Essas análises foram realizadas com um microscópio óptico Zeiss modelo Axiophoto.

\subsection{Ensaios de Tração Iniaxial}

Os corpos de prova para os ensaios de tração uniaxial foram confeccionados no Centro de Pesquisas da CSN, com utilização de prensa hidráulica com punção e matriz segundo as dimensões da norma NBR 6673.(4) Os ensaios foram realizados em uma máquina Instron 4206 , em dois corpos de prova, conforme orientação de $0,45^{\circ}$ e $90^{\circ}$, em relação ao sentido de laminação, para obtenção de propriedades mecânicas, por exemplo, limite de escoamento, limite de resistência, alongamento total, expoente de encruamento e coeficiente de anisotropia plástica.

\subsection{Previsões Teóricas de Deformações Limites}

A curva definida pelos eixos das menores $\left(\varepsilon_{2}\right)$ e maiores $\left(\varepsilon_{1}\right)$ deformações principais, obtidas no plano da chapa por meio de trajetórias lineares de deformação, é conhecida como Curva Limite de Conformação. ${ }^{(5,6)}$ A CLC pode ser definida ora na condição de estricção localizada ora na fratura. ${ }^{(7)}$ No domínio de estampagem $\left(\varepsilon_{2} \leq 0, \varepsilon_{1}>0\right)$ é possível adotar o critério de Hill( ${ }^{(8)}$ que estabelece que a instabilidade plástica ocorre quando a taxa de encruamento, associada com um estado plano de tensões principais $\left(\sigma_{1}, \sigma_{2}\right)$, iguala-se à taxa de amolecimento geométrico. Considera-se um carregamento proporcional, no qual as razões entre deformações plásticas principais $\left(\rho=\varepsilon_{2} / \varepsilon_{1}\right)$ e tensões principais $\left(\alpha=\sigma_{2} / \sigma_{1}\right)$ são constantes. $O$ comportamento plástico do material em tração uniaxial pode ser descrito pela equação de Holloman $\sigma=K \varepsilon^{n}$, onde $K$ é o coeficiente de resistência e $\mathrm{n}$ é o expoente de encruamento. Hill ${ }^{(8)}$ considera que a estricção localizada se desenvolve ao longo de uma banda inclinada com respeito à maior tensão principal, e que 
sua orientação se desenvolve ao longo da direção de um estado plano de deformação. Nesta condição, as previsões de deformações limites do critério de Hill( ${ }^{(8)}$ fornecem:

$$
\begin{aligned}
& \varepsilon_{1}^{*}=\frac{n}{I+\rho} \\
& \varepsilon_{2}^{*}=\frac{n \rho}{I+\rho}
\end{aligned}
$$

As previsões do critério de Hill(8) descrevem uma reta no diagrama de deformações principais $\left(\varepsilon_{2}, \varepsilon_{1}\right)$ na região de estampagem da CLC entre o estado de tração uniaxial, $\left(\varepsilon_{2}, \varepsilon_{1}\right)=(-n, 2 n)$, e o estado de deformação plana, $\left(\varepsilon_{2}, \varepsilon_{1}\right)=(0, n)$. Ademais, no estado de tração uniaxial a razão de deformações plásticas $\rho=\varepsilon_{2} / \varepsilon_{\text {, é relacionada }}$ com o coeficiente de anisotropia plástica normal $\mathrm{R}$ por $\rho=-(R /(I+R))$. Uma estimativa para o estado de deformação plana é proposta por Keeler-Brazier ${ }^{(9)}$, em função da espessura inicial t e do expoente de encruamento $n$ :

$$
e_{1 z}=(23,30+\mid 4,13 t) \frac{n}{0,21}(\%)
$$

\subsection{Ensaios de Expansão Mecânica}

Os ensaios de expansão mecânica foram realizados em máquina universal de embutimento Erichsen, na qual foi realizada adaptação do dispositivo apresentado na Figura la. O sistema hidráulico dessa máquina aciona o ferramental de expansão, que possui dois cones em seu eixo central, que transferem o movimento axial em deslocamento radial das pinças. Foram desenvolvidos quatro conjuntos de ferramentas, com perfis de diferentes raios meridionais $(5 \mathrm{~mm}, 10 \mathrm{~mm}, 20 \mathrm{~mm}, 30 \mathrm{~mm}, 40 \mathrm{~mm}$, $50 \mathrm{~mm}, 60 \mathrm{~mm}$ e $70 \mathrm{~mm}$ ) e um com trecho em paralelo, podendo assim obter-se, após o processo de expansão mecânica, deformações circunferenciais positivas e meridionais negativas. A Figura Ib apresenta o desenho mecânico de uma pinça de expansão.

Todos os conjuntos de ferramentas propostos neste trabalho apresentam as seguintes constantes:

- diâmetros de abertura radial inicial e máxima final;
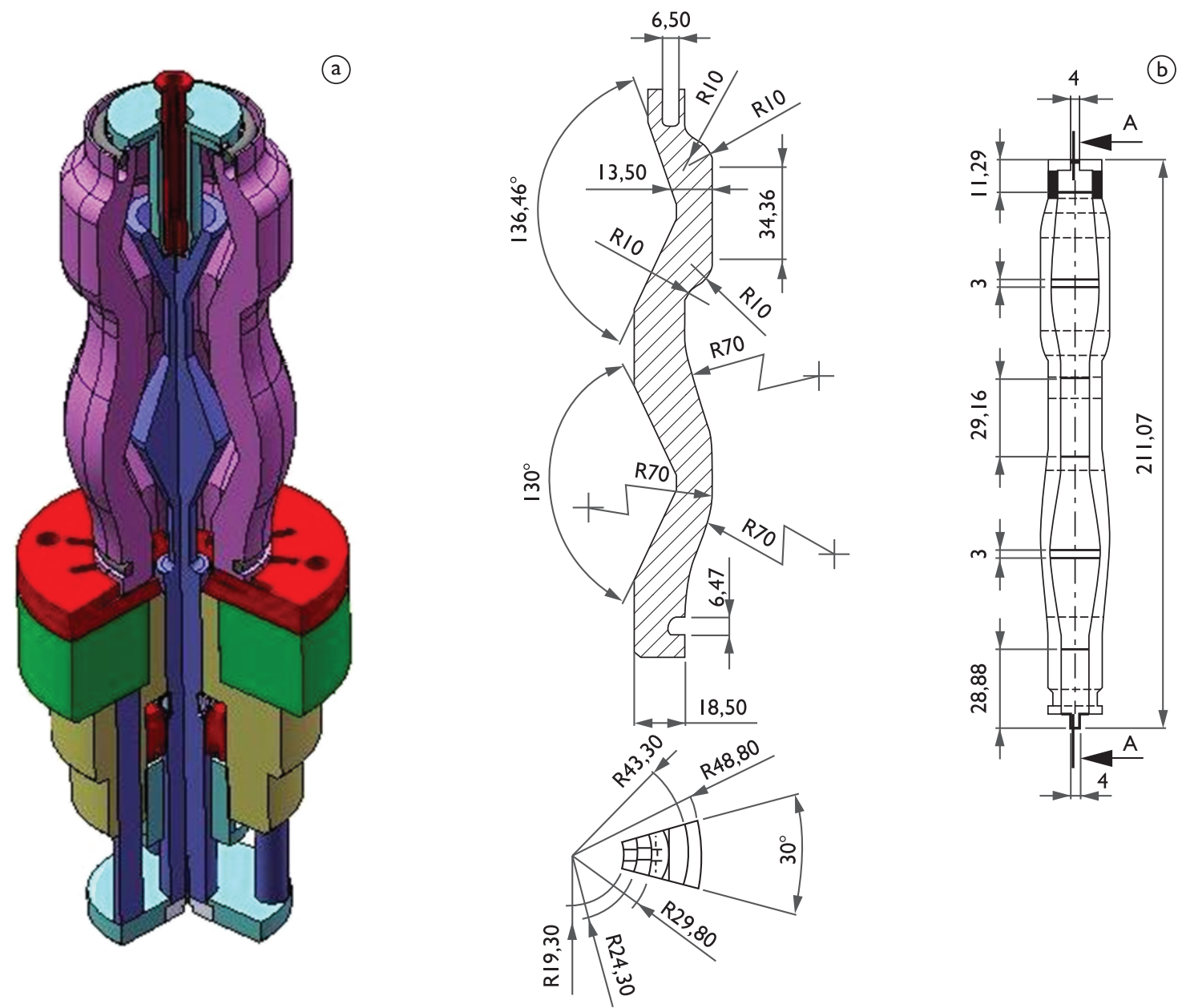

Figura I. Detalhes do dispositivo de expansão mecânica de tubos: a) conjunto do ferramental; e b) pinça de expansão com trecho em paralelo e raio de $70 \mathrm{~mm}$. 


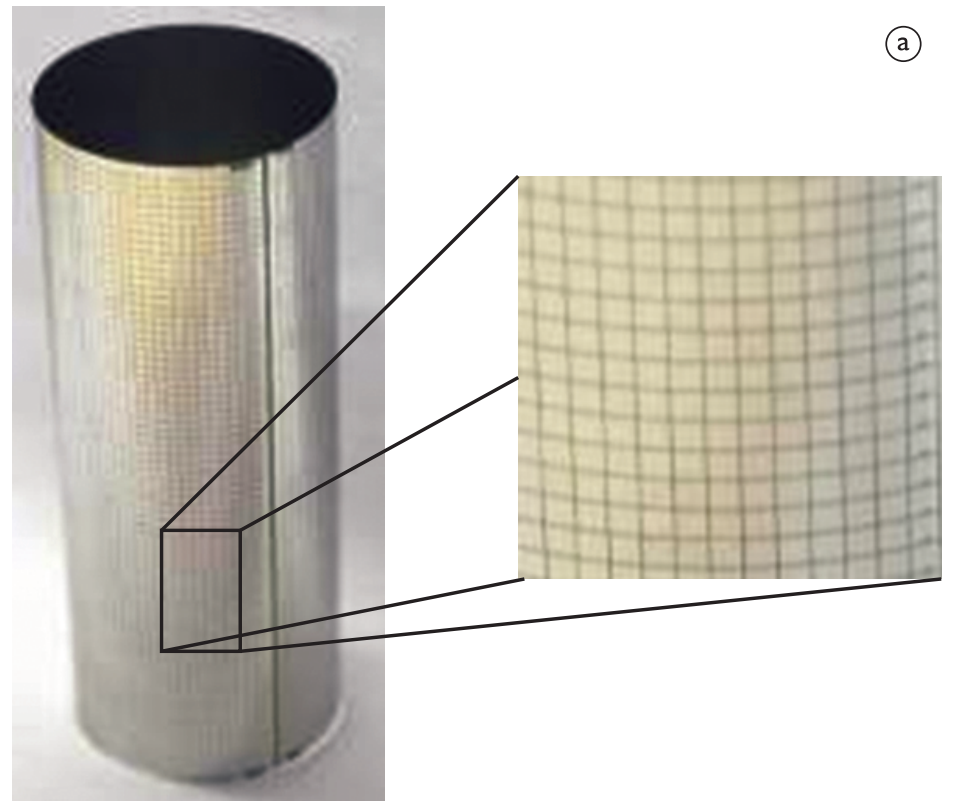

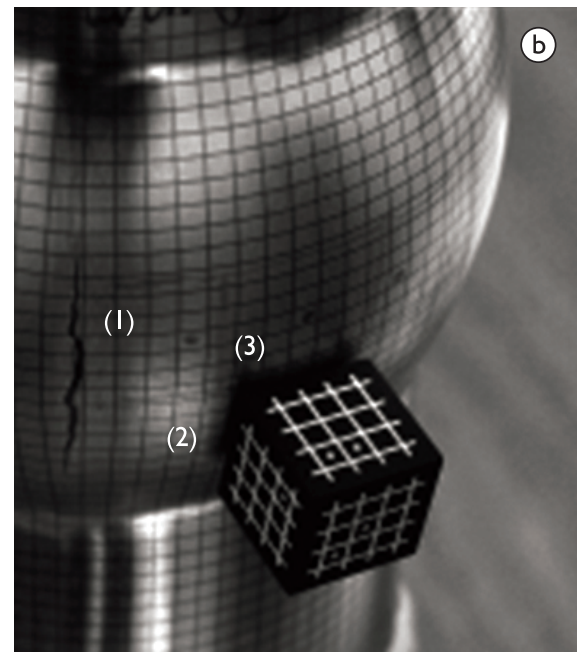

Regiōes de medidas

(I) Vizinho a fratura

(2) Superfície da pinça

(3) Vão entre pinças

Figura 2. Corpo de prova para ensaio limites de expansão mecânica de latas de três peças: a) detalhe de grade quadrada de 2,5 mm; e b) Corpo de prova após expansão com ferramental de raio $60 \mathrm{~mm}$.

- 12 pinças de expansão com mesma largura de contato; e

- rugosidade superficial média de $0,15 \mu \mathrm{m}$.

Os corpos de prova dos ensaios de expansão consistem em tubos soldados, confeccionados pela empresa Rimet, com diâmetro de $73 \mathrm{~mm}$ e altura de $174 \mathrm{~mm}$. Os tubos foram envernizados internamente com verniz epóxi incolor com camada seca de $5,8 \mathrm{~g} / \mathrm{m}^{2}$, e litografados no lado externo com grade quadrada de tamanho 2,5 mm, mostrada na Figura 2a. Para evitar rompimentos prematuros, o cordão de solda do tubo foi posicionado na superfície de uma das pinças. Em todos os ensaios os corpos de prova foram conduzidos até a ruptura. As deformações plásticas, segundo as direções circunferenciais $\left(\varepsilon_{1}\right)$ e meridionais $\left(\varepsilon_{2}\right)$, foram obtidas com o sistema de aquisição de imagens digitais e análise de deformações ASAME. ${ }^{(10)}$ As medidas de deformação foram realizadas em regiões adjacentes a fratura, nas regiões do corpo de prova referentes às superfícies das pinças e nos vãos entre as mesmas (Figura 2b).

\section{RESULTADOS E DISCUSSÃO}

Os resultados da composição química da folha de flandres em percentual de peso dos elementos químicos estão apresentados na Tabela I. Os resultados da análise de sanidade interna revelaram inclusões não metálicas do tipo óxido fino quantidade $1 / 2$. A Figura 3 apresenta a microestrutura composta por grãos ferríticos poligonais associados à cementita globular fina disposta em alinhamento, com o grão médio de 4,20 $\mu \mathrm{m}$ (I2,5 ASTM).
Tabela I. Composição química do aço

\begin{tabular}{cccccc}
\hline \multicolumn{6}{c}{ Elementos (\%) } \\
\hline $\mathbf{M n}$ & $\mathbf{P}$ & $\mathbf{S i}$ & $\mathbf{C u}$ & $\mathbf{N}$ & $\mathbf{C r}$ \\
\hline 0,2948 & 0,0226 & 0,0082 & 0,0034 & 0,0029 & 0,0083 \\
\hline $\mathbf{M o}$ & $\mathbf{C}$ & $\mathbf{S}$ & $\mathbf{B}$ & $\mathbf{T i}$ & $\mathbf{A l}$ \\
\hline$<0,000 \mathrm{I}$ & 0,0430 & 0,0100 & $<0,0001$ & $<0,000 \mathrm{I}$ & 0,0409 \\
\hline
\end{tabular}

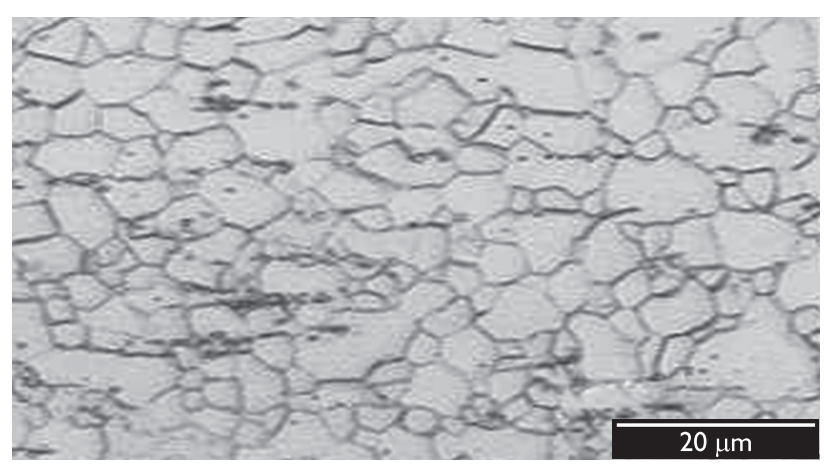

Figura 3. Microestrutura da folha de flandres de 0, $18 \mathrm{~mm}$.

A Tabela 2 apresenta os valores médios de propriedades mecânicas obtidas no ensaio de tração uniaxial, incluindo limite de escoamento a $0,2 \%$ de deformação plástica, $\sigma_{\mathrm{e}}$, limite de resistência, $\sigma_{r}$, expoente de encruamento, $n$, alongamento total, $A l \%$ e coeficiente de anisotropia plástica, R, definido em I2\% de deformação plástica. Os valores de anisotropia plástica normal e expoente de encruamento médio foram calculados por $\bar{R}=\left(R_{0}+2 R_{45}+R_{90}\right) / 4=0,70 \mathrm{e}$ 
$\overline{\mathrm{n}}=\left(\mathrm{n}_{0}+2 \mathrm{n}_{45}+\mathrm{n}_{90}\right) / 4=0,126$. Ademais, as deformações limites $\left(\varepsilon_{1} ; \varepsilon_{2}\right)$ em tração uniaxial e deformação plana previstas pelo critério de Hill ${ }^{(8)}$ com os valores de $\bar{n}$ e $\bar{R}$, Equações $(I-2)$, são iguais a $(0,2 \mid 4 ;-0,088)$ e $(0, \mid 26 ; 0)$, respectivamente. A previsão obtida com 0 modelo de Keeler-Brazier para o estado de deformação plana é $(0, I 44 ; 0)$.

Quanto aos resultados dos ensaios de expansão mecânica realizados em corpos de prova na forma de

Tabela 2. Propriedades mecânicas da folha de flandres de $0,18 \mathrm{~mm}$

\begin{tabular}{cccccc}
\hline $\begin{array}{c}\text { Orientação } \\
\left({ }^{\circ}\right)\end{array}$ & $\begin{array}{c}\sigma_{\mathrm{e}} \\
(\mathbf{M P a})\end{array}$ & $\begin{array}{c}\sigma_{\mathrm{r}} \\
(\mathbf{M P a})\end{array}$ & $\mathbf{n}$ & $\mathbf{A l \%}$ & $\mathbf{R}$ \\
\hline 0 & 423 & $45 \mathrm{I}$ & 0,127 & 17,2 & 0,71 \\
45 & 419 & 446 & 0,126 & 15,4 & 0,65 \\
90 & 418 & 434 & 0,123 & 17,8 & 0,79 \\
\hline
\end{tabular}

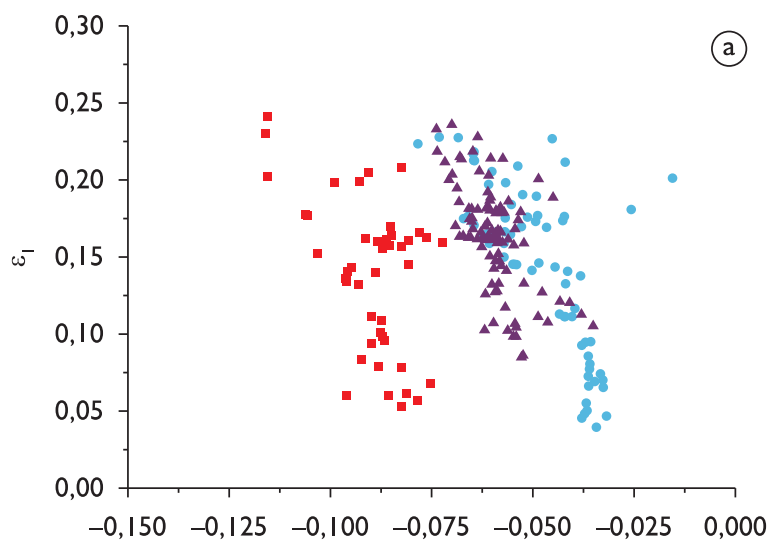

$\varepsilon_{2}$

- Raio $5 \mathrm{~mm}$ Raio $10 \mathrm{~mm} \Delta$ Raio $20 \mathrm{~mm}$ tubos soldados, foi observado, em todas as geometrias de ferramenta, que as fraturas nos corpos de prova ocorrem nos vãos localizados entre as pinças. Essa característica era esperada em razão das forças de atrito entre a superfície interna do tubo com as ferramentas de expansão. $\mathrm{Na}$ verdade, a restrição ao movimento circunferencial devido ao contato impõe maior deformação em regiões do tubo localizadas nos vãos entre as pinças de expansão. Para realizar a síntese de análise dos resultados, são apresentados, na Figura 4, os valores de deformações $\left(\varepsilon_{1} ; \varepsilon_{2}\right)$ divididos em três grupos de ferramentas. $\mathrm{Na}$ Figura $4 \mathrm{a}$ encontra-se a geometria com raio de $5 \mathrm{~mm}$, que proporciona maior severidade de expansão mecânica com deformações plásticas na ordem de $\varepsilon_{1}=0,24 \mathrm{e}$ $\varepsilon_{2}=-0,12$. Já as ferramentas com raios iguais a $10 \mathrm{~mm}$ e $20 \mathrm{~mm}$ apresentam resultados próximos.

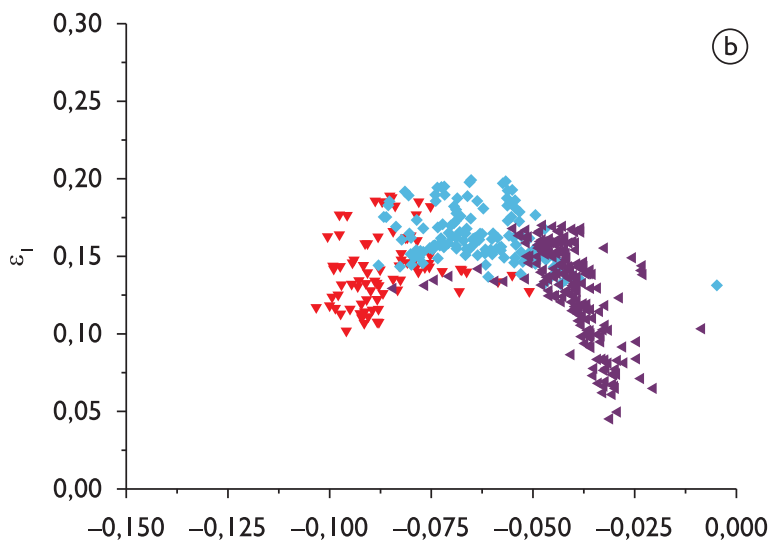

$\varepsilon_{2}$

$\nabla$ Raio $30 \mathrm{~mm}$ \& Raio $40 \mathrm{~mm} \triangleleft$ Raio $50 \mathrm{~mm}$

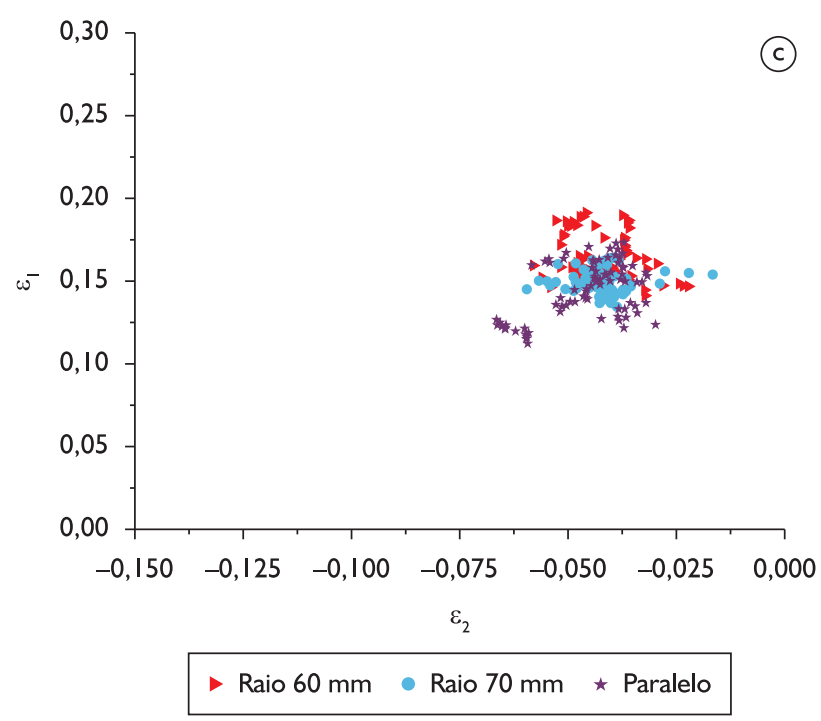

Figura 4. Deformações principais resultantes de ensaios de expansão em função do ferramental: a) raios $5 \mathrm{~mm}, 10 \mathrm{~mm}$ e $20 \mathrm{~mm}$; b) raios $30 \mathrm{~mm}, 40 \mathrm{~mm}$ e $50 \mathrm{~mm}$; e c) raios $60 \mathrm{~mm}$ e $70 \mathrm{~mm}$ e ferramenta paralela. 
No segundo grupo é possível observar mudanças nas trajetórias de deformação e decréscimo no nível da menor deformação com o aumento do raio da ferramenta (vide valores para raio de $50 \mathrm{~mm}$ na Figura 4b).

No último grupo (Figura 4c), a ferramenta com raio de $60 \mathrm{~mm}$, forneceu maior nível da deformação principal $\varepsilon_{1}$ em comparação às ferramentas com raio de $70 \mathrm{~mm}$ e trecho paralelo. Em todos os resultados obtidos é observada uma grande dispersão de valores, em razão da depuração de deformações ter sido realizada tanto em pontos de regióes vizinhas a fratura do corpo de prova e regiões referentes à superfície de pinça e vãos entre pinças. Ademais, algumas geometrias de ferramenta forneceram valores próximos de deformações.

No sentido de estabelecer a Curva Limite de Expansão (CLE) para qualificação de folhas metálicas destinadas à conformação de embalagens de três peças, são considerados somente os pontos simétricos situados em ambos os lados da fratura para definição de valores médios das deformações plásticas principais $\left(\varepsilon_{1} ; \varepsilon_{2}\right)$. A Figura 5 apresenta os resultados depurados na forma de uma Curva Limite de Expansão (CLE), na qual foram acrescentados os ajustes lineares obtidos a partir de previsões com o critério de Hill(8) em conjunto com a equação proposta por Keeler e Brazier ${ }^{(9)}$ para o estado plano de deformação. Primeiramente, observa-se uma tendência linear dos pontos experimentais que apresentam menor dispersão em comparação com as Figuras 4a e b. Em segundo lugar, o ajuste linear obtido a partir de previsões teóricas apresenta a mesma tendência que os valores experimentais. Contudo, estas previsões superestimam as deformações limites, em razão talvez da desconsideração dos efeitos das trajetórias de deformação que se encontram entre os estados de tração uniaxial e deformação plana.

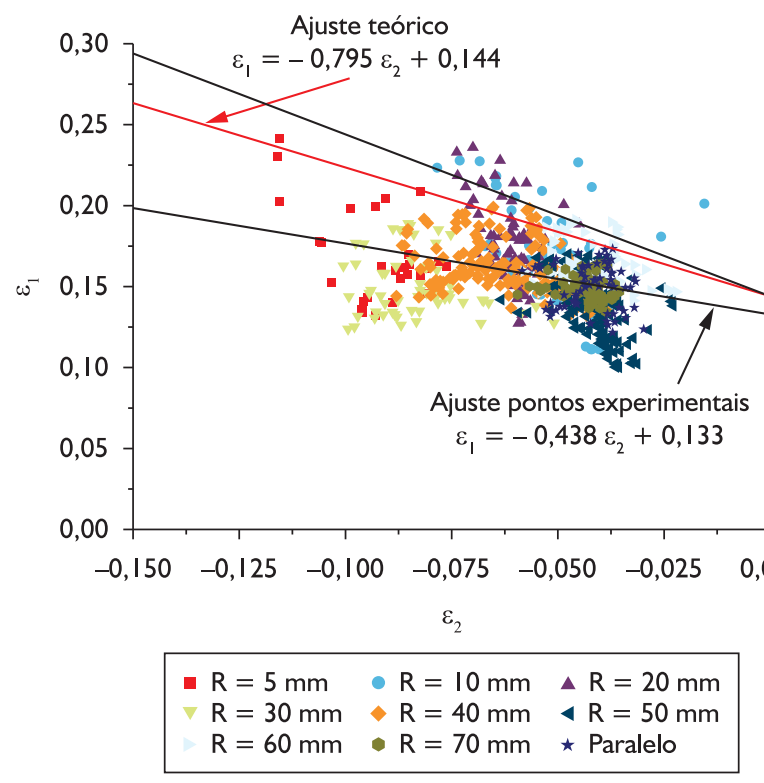

Figura 5. Curva Limite de Expansão (CLE) da folha de flandres de $0,18 \mathrm{~mm}$.
O dispositivo de expansão mecânica proposto neste trabalho é composto de nove ferramentas de ensaios de fácil manuseio, porém ainda carece de técnica refinada para obtenção de deformações a fim de garantir menor dispersão e maior reprodutibilidade. Recentemente, Pepelnjak e Barisic ${ }^{(11)}$ propuseram modificações nos desenhos de corpos de prova para obtenção da CLC de folhas de flandres de simples redução empregando punção plano do tipo Marciniak. ${ }^{(2)}$ Esses autores adotaram cinco amostras para cada largura, num total de 30 ensaios, para determinar a CLC de uma folha de flandres de $0.24 \mathrm{~mm}$ de espessura. As deformações foram depuradas a partir de imagens digitais obtidas durante $\circ$ ensaio, o que possibilitou determinar as trajetórias de deformação. Para aprimorar e explorar a técnica de expansão mecânica proposta neste trabalho, sugere-se primeiro realizar simulações por elementos finitos com objetivo de prever as trajetórias de deformações, visando otimizar a geometria dos ferramentais. Em seguida, propõe-se adotar o método de depuração definido pela norma ISO $12004^{(13,14)}$ para minimizar a dispersão dos valores de deformações limites.

\section{CONCLUSÕES}

Neste trabalho é proposto um novo método de avaliação da capacidade de expansão mecânica de folhas metálicas destinadas a embalagens de três peças. A partir das análises experimentais e teóricas, realizadas para uma folha de flandres de $0,18 \mathrm{~mm}$, é possível estabelecer as seguintes conclusões:

- o material avaliado apresentou boa homogeneidade de propriedades mecânicas, das características microestruturais, da sanidade interna e anisotropia plástica condizentes com - baixo teor de carbono e processamento termomecânico de folhas metálicas. O material também apresentou elevado nível de deformação por expansão mecânica superando aquele obtido a partir do ensaio de tração uniaxial;

- as ferramentas projetadas para os ensaios de expansão mecânica apresentaram excelente estabilidade, e resultados que reproduzem os estados de deformação presentes nos processos de fabricação de embalagens metálicas por expansão; e

- a metodologia proposta é de fácil execução e não apresenta nenhuma limitação relacionada com a baixa espessura do esboço, sobretudo com respeito a rupturas prematuras observadas normalmente nas técnicas convencionais de determinação da $\operatorname{CLC}^{(2,3)}$ e, portanto, pode tornar-se muito útil na concepção de novas embalagens metálicas atendendo às novas tendências de mercado. 


\section{Agradecimentos}

Os autores agradecem à Companhia Siderúrgica Nacional - CSN, e à empresa Rimet/RJ, que realizou a litografia e soldagem dos corpos de prova. Luciano Pessanha
Moreira agradece à Fundação de Amparo à Pesquisa do Estado do Rio de Janeiro - FAPERJ, pelo apoio financeiro, e ao Conselho Nacional de Desenvolvimento Científico e Tecnológico - CNPq, pela Bolsa de Produtividade em Pesquisa.

\section{REFERÊNCIAS}

I BRASIL Pack Trends 2005: embalagem; distribuição e consumo. 2. ed. Campinas: Instituto de Tecnologia de Alimentos, 2000.

2 ASTM INTERNATIONAL. ASTM E45: 10el Standard test methods for determining the inclusion content of steel. West Conshohocken, 2010. http://dx.doi.org/I0.1520/E0045-IOEOI

3 ASTM INTERNATIONAL. ASTM EI /2: I0 Standard test methods for determining average grain size. West Conshohocken, 2010. http://dx.doi.org/10.1520/EOI I2-10

4 ASSOCIAÇÃO BRASILEIRA DE NORMAS TÉCNICAS. NBR 6673: Produtos planos de aço: determinação das propriedades mecânicas a tração: método de ensaio. Rio de Janeiro, |98|.

5 KEELER, S. P. Determination of the forming limits in automotive stamping. Sheet Metal Industries, v. 42, n. 46I, p. 683-9I, Sept. 1965.

6 GOODWIN, G. M. Application of the strain analysis to sheet metal forming in the press shop. In: SAE AUTOMOTIVE ENGINEERING CONGRESS, 1968, Detroit, USA. [S.n.t.]. SAE paper 680093.

7 LUIZ, V. D.; CAMPOS, H. B.; MELO, T. M. F. Curva limite de estampagem à fratura (CLEF) versus curva limite de estampagem à estricção (CLEE). In: CONGRESSO ANUAL DA ABM, 62, 2007, Vitória, ES. São Paulo: ABM, 2007. p. 44-5I.

8 MARCINIAK, Z.; DUNCAN, J. L.; HU, S. J. Mechanics of sheet metal forming. Reino Unido: Butterworth-Heinemann, 2002.

9 KEELER, S. P.; BRAZIER; W. G. Relationship between laboratory material characterization and press shop formability. In: MICROALLOYING 75: INTERNATIONAL SYMPOSIUM ON HIGH STRENGTH LOW ALLOY STEELS. 1975, Washington, D.C. Proceedings... Metals Park; ASM, 1977. p. 517-28.

10 ASAME. Reference Manual Version 4.I2, Janeiro, 2008.

II PEPELNJAK, T.; BARISIC, B. Computer-assisted engineering determination of the formability limit for thin sheet metals by a modified Marciniak method. The Journal of Strain Analysis for Engineering Design, v. 44, n. 6, p. 459-72, Ago. 2009.

I2 MARCINIAK, Z.; KUCZYNSKI, K.; POKORA, T. Influence of the plastic properties of the material on the forming limit diagram for sheet metal tension. International Journal of Mechanical Sciences, v. 15, n. 10, p. 789-805, Oct. 1973. http://dx.doi.org/10.1016/0020-7403(73)90068-4

I 3 INTERNATIONAL ORGANIZATION FOR STANDARDIZATION. ISO I2004-2: Metallic materials-sheet and strip-determination of forming limit curves - part 2: determination of forming limit curves in laboratory. Geneva, 2007.

I 4 HOGSTROM, P.; RINGSBERG, J. W.; JOHNSON, E. An experimental and numerical study of the effects of length scale and strain state on the necking and fracture behaviours in sheet metals. International Journal of Impact Engineering, v. 36, n. I0-I I, p. II94-203, Oct.-Nov. 2009. http://dx.doi.org/I0. I0I6/j.ijimpeng.2009.05.005

Recebido em: 09/12/2010

Aceito em: 06/04/20II 